\title{
Highly Sensitive Local Surface Plasmon Resonance in Anisotropic Au Nanoparticles Deposited on Nanofibers
}

\author{
Masanari Saigusa, Kazuma Tsuboi, Yuichi Konosu, Minoru Ashizawa, \\ Akihiko Tanioka, and Hidetoshi Matsumoto \\ Department of Organic and Polymeric Materials, Tokyo Institute of Technology, 2-12-1 Ookayama, Meguro-ku, Tokyo 152-8552, Japan
}

Correspondence should be addressed to Hidetoshi Matsumoto; matsumoto.h.ac@m.titech.ac.jp

Received 19 December 2014; Accepted 14 February 2015

Academic Editor: Rizia Bardhan

Copyright (C) 2015 Masanari Saigusa et al. This is an open access article distributed under the Creative Commons Attribution License, which permits unrestricted use, distribution, and reproduction in any medium, provided the original work is properly cited.

\begin{abstract}
This paper reports the facile and high-throughput fabrication method of anisotropic Au nanoparticles with a highly sensitive local surface plasmon resonance (LPR) using cylindrical nanofibers as substrates. The substrates consisting of nanofibers were prepared by the electrospinning of poly(vinylidene fluoride) (PVDF). The Au nanoparticles were deposited on the surface of electrospun nanofibers by vacuum evaporation. Scanning electron microscopy revealed the formation of a curved Au island structure on the surface of cylindrical nanofibers. Polarized UV-visible extinction spectroscopy showed anisotropy in their LPR arising from the high surface curvature of the nanofiber. The LPR of the Au nanoparticles on the thinnest nanofiber with a diameter of $\sim 100 \mathrm{~nm}$ showed maximum refractive index (RI) sensitivity over $500 \mathrm{~nm} / \mathrm{RI}$ unit (RIU). The close correlation between the fiber diameter dependence of the RI sensitivity and polarization dependence of the LPR suggests that anisotropic Au nanoparticles improve RI sensitivity.
\end{abstract}

\section{Introduction}

In this decade, local surface plasmons (LPRs) in Au and Ag nanoparticles, which have been used in stained-glass in medieval European churches, have received a great deal of attention from researchers in optics, biochemistry, biogenetics, and medical science, due to their fascinating optical characteristics. One is their ability to enhance the light field intensity. The light field resonant with LPR is dramatically enhanced around the surface of the nanoparticle, which ultimately allows us to obtain an optical signal from single molecules. This enhancement effect is fairly effective in Raman scattering [1-5], fluorescence [6, 7], and nonlinear optical phenomena [8]. Another feature is the sensing ability of a very small amount of a dielectric substance. The resonance wavelength of the LPR sensitively varies with the dielectric condition in the surroundings of a nanoparticle. It has been applied to the transducer of a label-free biosensor that detects the affinity between biomaterials such as a virus, specific chemicals, DNAs, and proteins without fluorescent labeling [9-17]. These LPR characteristics can be utilized with a single nanoparticle $[18,19]$; then the nanoparticle with the LPR was often expected to be an in vivo nanosensor or an in vivo nanosensitizer detecting biochemical materials in living cells or animals $[20,21]$.

Recently, the focus on the LPR in anisotropic nanoparticles of $\mathrm{Au}$ and $\mathrm{Ag}$ has increased because of significant improvements in the LPR characteristics, representing a high sensitivity to refractive index (RI) variations. For example, the RI sensitivity of the LPR in an Au nanorod has been reported to be $150 \sim 200 \mathrm{~nm} /$ refractive index unit (RIU), while the sensitivity of a true spherical isotropic Au nanoparticle is 20 $50 \mathrm{~nm} / \mathrm{RIU}$ [22-24]. To prepare anisotropic nanoparticles, both chemical and physical methods have been exploited. For the main chemical one, small Au nanospheres coated with an amphiphilic surface agent have been reduced in $\mathrm{HAuCl}_{4}$ or $\mathrm{NaAuCl}_{4}$ solution, resulting in wide variety of anisotropic nanoparticles, such as nanorods, nanobipyramids, and nanostars [25]. For the physical ones, lithographic techniques using a focused ion beam or electron beam have been conducted for nanowires, nanodisks [26], and closely adjacent nanopillars [27]. Physical template methods with 


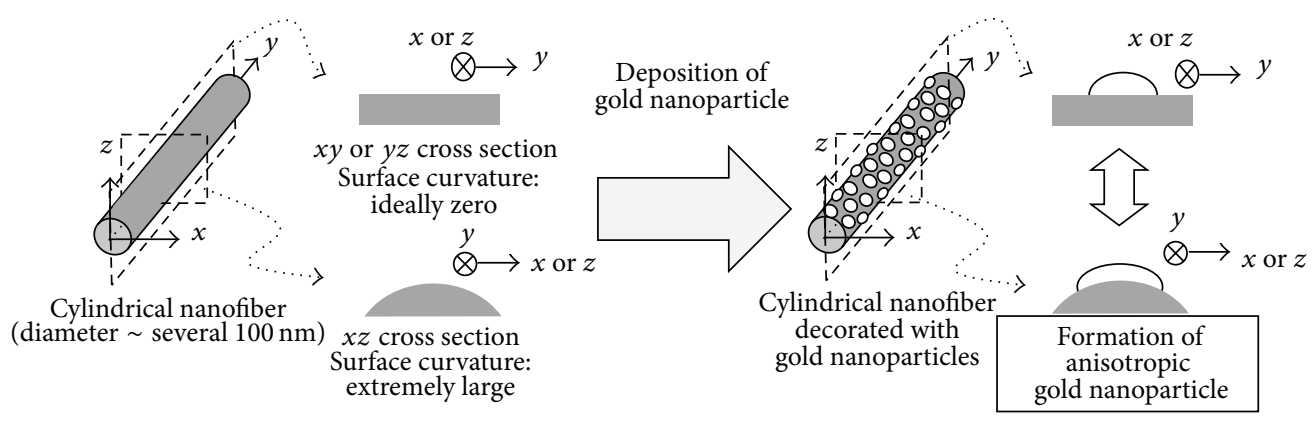

FIGURE 1: Schematic illustration of the fabrication of anisotropic Au nanoparticles using cylindrical nanofiber substrate.

polymer or silica nanospheres have also been employed for cap-shaped Au nanoparticles [28], nanotriangles [29, 30], and nanocrescents [31]. In addition to them, an $\mathrm{Au}$ island film structure formed by direct evaporation in a vacuum on a transparent substrate, glass slide, or indium tin oxide (ITO) glass shows a modest high sensitivity ( $200 \mathrm{~nm}$ RIU) [32]. However, these methods described above have some of disadvantages, low reproducibility and low throughput, the difficulty in locating the nanoparticles to the desired position with desired orientation for the chemical reduction method, high-cost for instruments for the lithographic techniques, and unavailability of the anisotropic nanoparticles with a high aspect ratio for highly sensitive LPR, for the physical template methods and the direct evaporation of Au or Ag.

In the present study, we used cylindrical surface of a nanofiber as a template substrate for the deposition of anisotropic nanoparticles. As illustrated in Figure 1, a cylindrical nanofiber has two kinds of surface curvatures. One is a high surface curvature in the cross-sectional plane (crosssection including $x$-axis and $z$-axis in Figure 1 ) and the other is an ideally zero surface curvature along the fiber axis ( $y$-axis) (cross-section including $y$-axis). There is an extremely large contrast between the two surface curvatures. We postulated that when the Au nanoparticles were deposited on the surface of the nanofiber, the contrast in the surface curvature is reflected in the shape of the Au nanoparticles and induces anisotropy, resulting in an enhanced RI sensitivity. In particular, the size of the Au nanoparticle is comparable to the fiber diameter as depicted in the right panel of Figure 1; thus their curving will be enhanced with a decrease in the fiber diameter (i.e., with an increase in the surface curvature). To prepare a cylindrical nanofiber, electrospinning has been adopted, since electrospinning is a simple and versatile method for producing thin fibers. Major advantages of electrospinning are (i) applicability for various materials such as synthetic and natural polymers, inorganic molecules, and composites and (ii) ability to produce thin fibers with well-controlled diameters in the micrometer and nanometer ranges [33-36]. The combination of the two simple processes, electrospinning and successive vacuum evaporation, will provide a high-throughput in the production of the anisotropic Au nanoparticles with a highly sensitive LPR on nanofibers, in other words, the hierarchystructured LPR sensing nanofiber.
We first prepared nanofibers of poly(vinylidene fluoride) (PVDF) on an ITO glass slide by electrospinning [37] as a supporting substrate. Second, anisotropic Au nanoparticles were vacuum-deposited onto the surface of the nanofibers. The aims of the present study are (i) to present a novel fabrication method of the anisotropic Au nanoparticles by electrospinning and successive vacuum deposition and (ii) to investigate the effect of the high surface curvature of nanofibers on the shape of the Au nanoparticles and the RI sensitivity of their LPR characteristics.

\section{Materials and Methods}

2.1. Materials. poly(vinylidene fluoride) (PVDF), $M_{w}=$ 275,000, was purchased from Sigma Aldrich. N, N-Dimethylacetamide (DMAc), pyridine, and glycerin were purchased from Wako Chemical. All reagents were used without further purification.

\subsection{Preparation of PVDF Nanofiber Substrate by Electro-} spinning. Three kinds of PVDF nanofibers as the substrates were prepared on an ITO glass slide by electrospinning, in which the mean diameters were controlled to about $500 \mathrm{~nm}, 200 \mathrm{~nm}$, and $100 \mathrm{~nm}$. PVDF was dissolved in DMAc at $25 \mathrm{wt} \%, 20 \mathrm{wt} \%$, and $17 \mathrm{wt} \%$, respectively. Pyridine was added to the spinning solutions as an organic electrolyte at a concentration of $5 \mathrm{wt} \%$ and $1 \mathrm{wt} \%$ (to solvent) to the $20 \mathrm{wt} \%$ and the $17 \mathrm{wt} \%$ PVDF solutions, respectively, for preparation of thinner fibers [38]. The electrospinning conditions for the nanofibers with the mean diameters $\phi$ of $\sim 500 \mathrm{~nm}, \sim 200 \mathrm{~nm}$, and $\sim 100 \mathrm{~nm}$ were as follows. For the electrospun nanofiber substrate of $\phi \sim 500 \mathrm{~nm}$, the applied voltage between the nozzle and the collector $V$ was $10 \mathrm{kV}$, the distance between the nozzle and the collector was $D=12 \mathrm{~cm}$, and the flow rate was, $\mathrm{FR}=5 \mu \mathrm{L} / \mathrm{min}$. For the nanofiber substrate of $\phi \sim 200 \mathrm{~nm}, V=20 \mathrm{kV}, D=12 \mathrm{~cm}$, and FR $=1 \mu \mathrm{L} / \mathrm{min}$, and for the nanofiber of $\phi \sim 100 \mathrm{~nm}, V=60 \mathrm{kV}, D=14 \mathrm{~cm}$, and $\mathrm{FR}=1 \mu \mathrm{L} / \mathrm{min}$. The residual solvent in the nanofibers was removed by vacuum evacuation for $3 \mathrm{~h}$. To prepare the spin-coated PVDF film, as a reference, several tens of $\mu \mathrm{L}$ of a PVDF/DMAc solution was dropped onto an ITO glass slide. The ITO glass slide was rotated for three minutes at $200 \mathrm{rpm}$ to prepare the PVDF spin-coated film. 
2.3. Formation of Au Nanoparticles by Vacuum-Evaporation. $\mathrm{Au}$ nanoparticles (i.e., isolated $\mathrm{Au}$ island structures) were directly vacuum-deposited on the prepared nanofiber substrates and the spin-coated film in a vacuum below 5.0 $\times 10^{-4} \mathrm{~Pa}$. The deposition rate was $0.10 \mathrm{~nm} / \mathrm{s}$. The deposition was continued until the quartz microbalance showed $4.0 \mathrm{~nm}$.

2.4. SEM Observations. The morphologies of the PVDF nanofibers were observed using scanning electron microscope (SEM: SM-200, Topcon). The accelerating voltage was $10 \mathrm{kV}$ and the working distance was fixed at $10 \mathrm{~mm}$. To avoid charging, the surface of nanofibers was sputter-coated with $\mathrm{Au}$. The morphology of the Au nanoparticles on the nanofiber substrate was observed by a backscattering electron image (BEI) using a field-emission SEM (FE-SEM: S-4500, Hitachi). As the light source, Nd-YAG laser was used. The accelerating voltage was $15 \mathrm{kV}$ and the working distance was $15 \mathrm{~mm}$. The BEI image samples were prepared on a small Si wafer and coated with carbon.

2.5. UV-Visible Extinction Spectroscopy. The UV-visible extinction spectra of the prepared samples were measured using optical fibers connected to a monochromator (USB4000: Ocean Optics), with a white light from a halogen light (LS1LL: Ocean optics). A beam of white light was incident to the sample surface through a beam collimator placed at the end of the optical fiber. The spot diameter of the incident beam was adjusted to about $1 \mathrm{~mm}$ on the surface of the sample by an iris when the beam angle of incidence $\theta$ was $0^{\circ}$. For the reference of measurement, the parts of the nanofiber substrates without Au nanoparticles were used. The polarized extinction spectra of the samples were measured with a Glan prism located before the sample. The electric field of the incident light was controlled to be $\mathrm{p}$ - or s-polarization with respect to the sample surface. The angle of incidence was changed using a rotational stage to $\theta=0^{\circ}, 30^{\circ}, 50^{\circ}$, and $70^{\circ}$. For evaluating the RI sensitivity of the LPR, the sample was immersed into various RI liquids $(n=1.3330 \sim 1.4050)$ in a cuvette, and its extinction spectra were measured at $\theta=0^{\circ}$. Each liquid was prepared by mixing water and glycerin to the appropriate ratio. The RI of the liquid was determined with a refractometer. The RI sensitivity of the LPR was evaluated in terms of the RIU from the RI dependence of the LPR extinction peak wavelength.

\section{Results and Discussion}

3.1. Morphologies of Anisotropic Au Nanoparticles Deposited on Nanofibers. The morphologies of the three kinds of prepared electrospun nanofibers were first observed by SEM. The bead-free, smooth, and cylindrical fibers were obtained. Their mean fiber diameters were $\phi=489 \pm 106 \mathrm{~nm}, 211 \pm 43 \mathrm{~nm}$, and $114 \pm 32 \mathrm{~nm}$. The Au nanoparticle samples, vacuum-deposited on these nanofibers, were called AuNF500, AuNF200, and AuNF100, in the order of mean fiber diameter, respectively. The Au nanoparticle prepared on the spin-coated PVDF film was called AuSPF.
The overhead views of the prepared Au nanoparticles were observed by BEI observation using FE-SEM. Figures 2(a), 2(b), 2(c), and 2(d) show the BEIs of AuNF500, AuNF200, AuNF100, and AuSPF, respectively. In all the prepared samples, the $\mathrm{Au}$ nanoparticles form island-type structures [33-35]. In Figure 2(c), there are 3-5 Au nanoparticles on the width of the nanofiber of $\sim 100 \mathrm{~nm}$ in diameter. This image clearly showed that the prepared Au nanoparticles were curved along with the cylindrical surface of the nanofiber. On the $\sim 200 \mathrm{~nm}$ and $\sim 500 \mathrm{~nm}$ diameter nanofibers (Figures 2(a) and 2(b)), there are 8-10 and over $20 \mathrm{Au}$ nanoparticles on their widths, respectively. In the samples, the surfaces of the nanofiber without any $\mathrm{Au}$ nanoparticles formed by masking of the Au vapor beam with the neighboring nanofibers. The formation of these parts supported that the Au vapor beam did not go behind the nanofibers, and the $\mathrm{Au}$ nanoparticles were deposited only on the front surfaces of the nanofibers. Though it is difficult to quantitatively characterize island structures like this, the overhead views of the Au nanoparticles were characterized in terms of the mean length of the major axis and the aspect ratio of the island structure with elliptical fitting. The obtained values are listed in Table 1. The size and the aspect ratio of $\mathrm{Au}$ nanoparticle increased with the decrease in the mean fiber diameter. This implies the contribution from the high-surface curvatures to the shape of $\mathrm{Au}$ nanoparticles while this analysis includes errors due to the principle of BEI and image distortion by the defocusing of curved surface, even though measuring the $\mathrm{Au}$ nanoparticles around the center of nanofiber.

3.2. LPR of Anisotropic Au Nanoparticles Deposited on Nanofibers. The LPR extinction spectra of all the prepared samples showed broad extinctions centered at $\lambda=530-$ $650 \mathrm{~nm}$ that are typical characteristics of the LPR in the $\mathrm{Au}$ island structure $[32,39]$, which agreed with the results of the SEM observations described above. Figure 3 shows the p- and s-polarized LPR extinction spectra of AuNF100 and AuSPF. The differences in the peak wavelength of the LPR extinctions, $\lambda_{\text {LPR }}$, and their widths would be due to a decreased Au thickness in the AuNF100 compared with those in the AuSPF.

The angle dependency of $\lambda_{\mathrm{LPR}}$ found in Figure 3(a) indicates the anisotropy of Au nanoparticles between the height and the mean size of the overhead view. Note that the AuNF100 sample (where Au nanoparticles were deposited on nanofibers) was characterized by polarized extinction spectroscopy the same as with the AuSPF sample (where Au nanoparticles were deposited on the flat substrate), since the Au nanoparticles deposited only on the front surface of nanofibers as found in our SEM observation. In Figure 3(a), the peak wavelength of the LPR extinction in the $\mathrm{p}$-polarized spectra, $\lambda_{\text {LPR }}(\mathrm{p})$, was modestly blue-shifted with the increase in $\theta$ while the peak wavelength in the s-polarized spectra, $\lambda_{\text {LPR }}(\mathrm{s})$, only slightly varied with $\theta$ (Figure $3(\mathrm{~b})$ ). At the grazing angle of $\theta=70^{\circ}$, the difference between $\lambda_{\mathrm{LPR}}(\mathrm{p})$ and $\lambda_{\mathrm{LPR}}(\mathrm{s}), \Delta \lambda_{\mathrm{LPR}}=\lambda_{\mathrm{LPR}}(\mathrm{s})-\lambda_{\mathrm{LPR}}(\mathrm{p})$, was $33 \mathrm{~nm}$. These behaviors of $\lambda_{\mathrm{LPR}}(\mathrm{p})$ and $\lambda_{\mathrm{LPR}}(\mathrm{s})$ clearly indicate that there are two modes in the LPR of the Au nanoparticles due to 


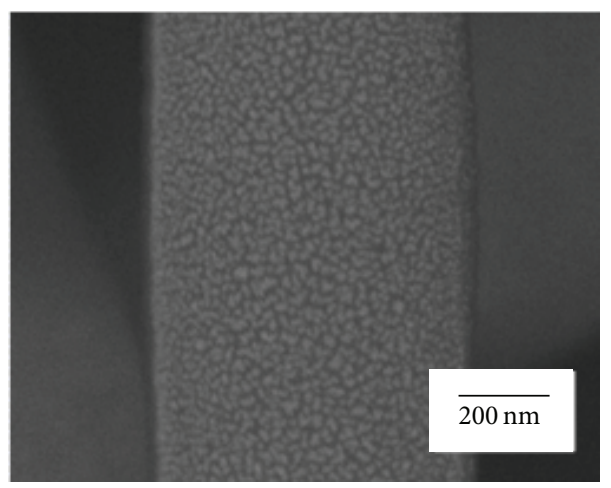

(a)

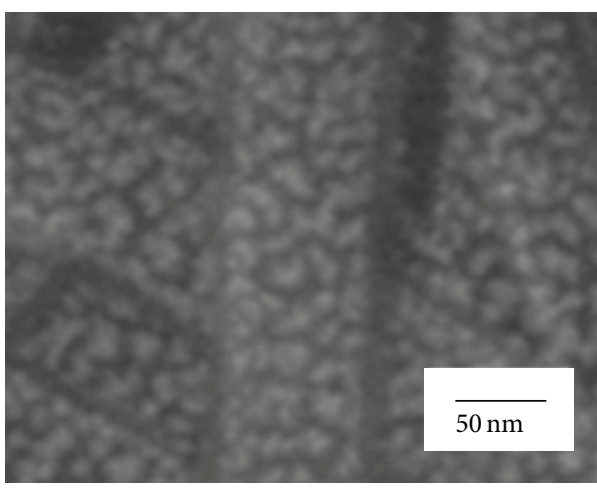

(c)

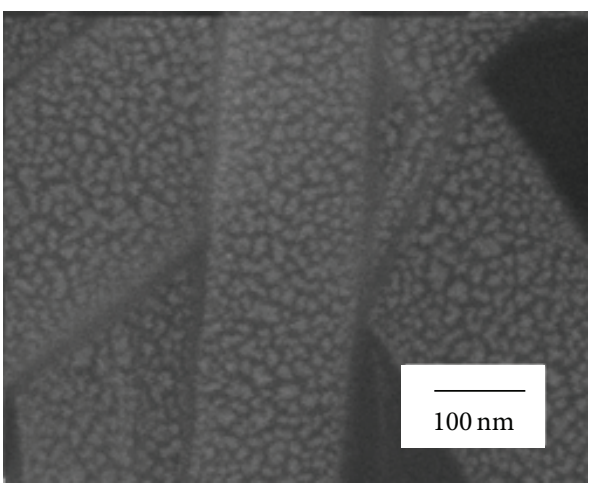

(b)

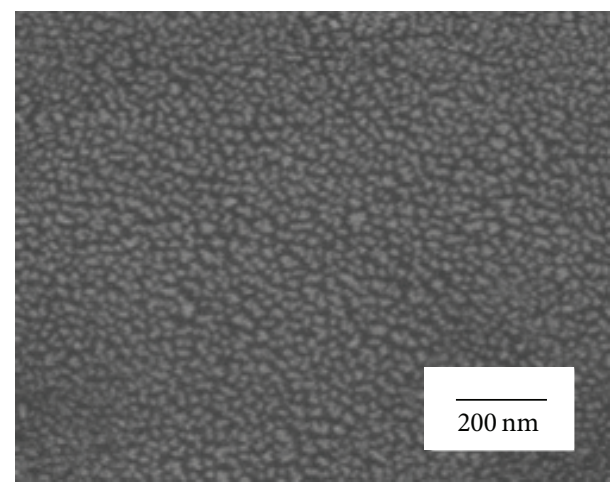

(d)

FIgURE 2: Backscattering electron images of the prepared Au nanoparticles. (a) AuNF500, (b) AuNF200, (c) AuNF100, and (d) AuSPF.

TABLE 1: The results from SEM image analysis.

\begin{tabular}{lccc}
\hline Sample & $\begin{array}{c}\text { Mean diameter of } \\
\text { substrate } \\
\text { nanofibers/nm }\end{array}$ & $\begin{array}{c}\text { Mean length of major } \\
\text { axis of } \\
\text { nanoparticles/nm }\end{array}$ & $\begin{array}{c}\text { Mean aspect ratio of } \\
\text { nanoparticles/nm }\end{array}$ \\
\hline AuSPF & $489 \pm 106$ & $18.8 \pm 5.7$ & $1.71 \pm 0.50$ \\
AuNF500 & $211 \pm 43$ & $20.9 \pm 8.1$ & $1.81 \pm 0.54$ \\
AuNF200 & $114 \pm 32$ & $20.4 \pm 6.6$ & $1.86 \pm 0.58$ \\
AuNF100 & & $22.1 \pm 8.5$ & $1.91 \pm 0.68$ \\
\hline
\end{tabular}

anisotropy, such as the mode along with major axis and that along with minor axis in the Au spheroid [39, 40], since, from the Mie's theory, the values of $\lambda_{\mathrm{LPR}}$ and $\Delta \lambda_{\mathrm{LPR}}$ in this geometry mainly depend on the aspect ratio between the height and the mean lateral size of the $\mathrm{Au}$ nanoparticles. According to the theoretical calculations by Warmack and Humphrey [39], $\Delta \lambda_{\text {LPR }}=33 \mathrm{~nm}$ with a dielectric substrate roughly corresponds to the aspect ratio of 2.0 2.2 for the shape of the Au nanoparticles. On the contrary, for AuSPF, a small variation was found, $\Delta \lambda_{\mathrm{LPR}}=7.8 \mathrm{~nm}$ (Figures $3(\mathrm{c})$ and $3(\mathrm{~d}))$, corresponding to the aspect ratio of around 0.8-1.0. This difference in the polarization dependence of $\lambda_{\text {LPR }}$ between AuNF100 and AuSPF indicates the different shape, aspect ratio between height and lateral size, of the $\mathrm{Au}$ nanoparticles. Taking into account the information from the overhead view by the SEM observation which shows relatively small variation in lateral shape and the difference in the aspect ratio, the Au nanoparticles in AuNF100 would be thinner than those in the AuSPF and slightly elongated.

The magnitude of $\Delta \lambda_{\mathrm{LPR}}$ could be a guideline for the anisotropy of the Au nanoparticles between the mean lateral size and height. In Table 2 , the $\Delta \lambda_{\text {LPR }}$ values obtained from the polarized spectra of all the prepared samples are listed. It can be seen that $\Delta \lambda_{\mathrm{LPR}}$ increased with a decrease in the mean diameter of the nanofibers from $\Delta \lambda_{\mathrm{LPR}}=8.0 \mathrm{~nm}$ to $33 \mathrm{~nm}$. The similar values of $\Delta \lambda_{\mathrm{LPR}}$ for AuNF500 $\left(\Delta \lambda_{\mathrm{LPR}}=8.0 \mathrm{~nm}\right)$ and $\operatorname{AuSPF}\left(\Delta \lambda_{\mathrm{LPR}}=7.8 \mathrm{~nm}\right)$ indicate small aspect ratio in them. For AuNF200 and AuNF100, $\Delta \lambda_{\text {LPR }}$ was over $20 \mathrm{~nm}$ and greater than the others, which indicates larger anisotropy between lateral size and height of $\mathrm{Au}$ nanoparticles than that in others. This finding may arise from thinning of the $\mathrm{Au}$ nanoparticles as described above. The fiber diameter dependence of the shape of the Au nanoparticles is well consistent with our expectation; that is, the cylindrical surface 

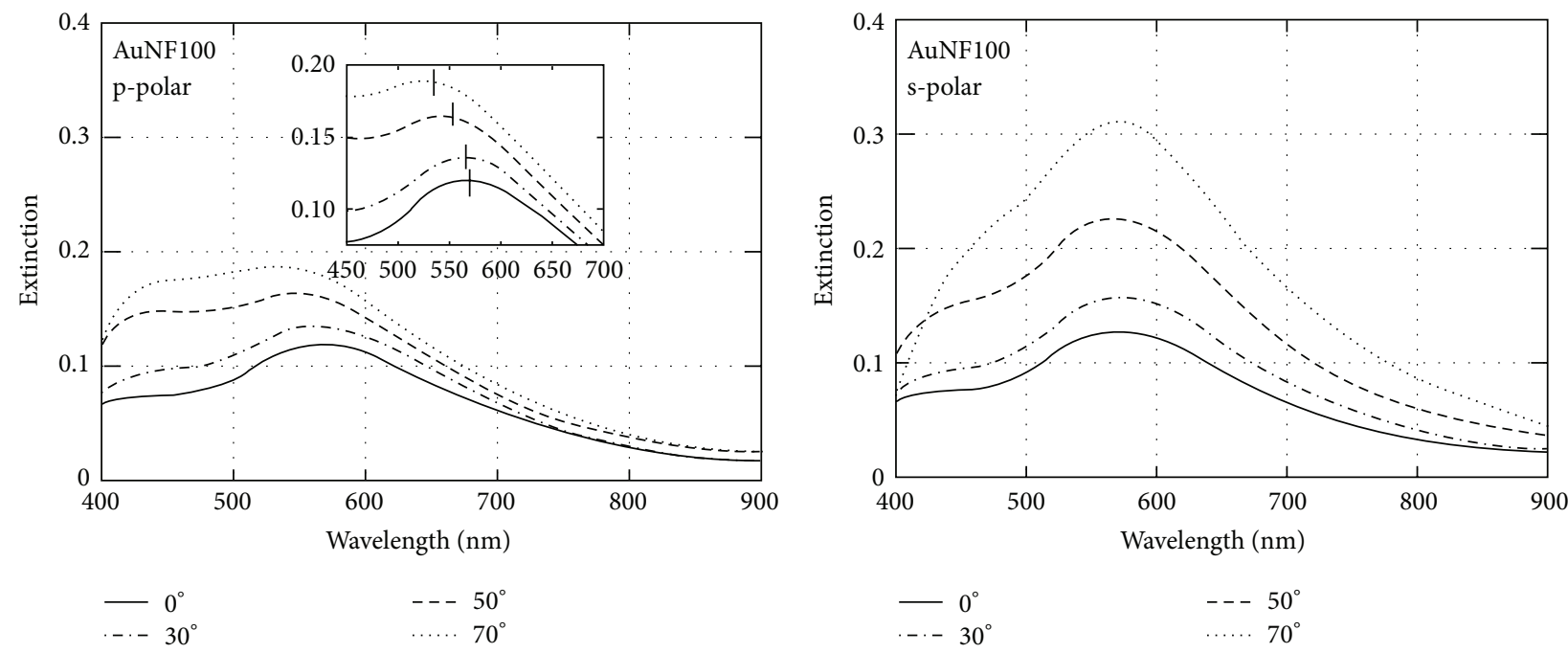

(a)

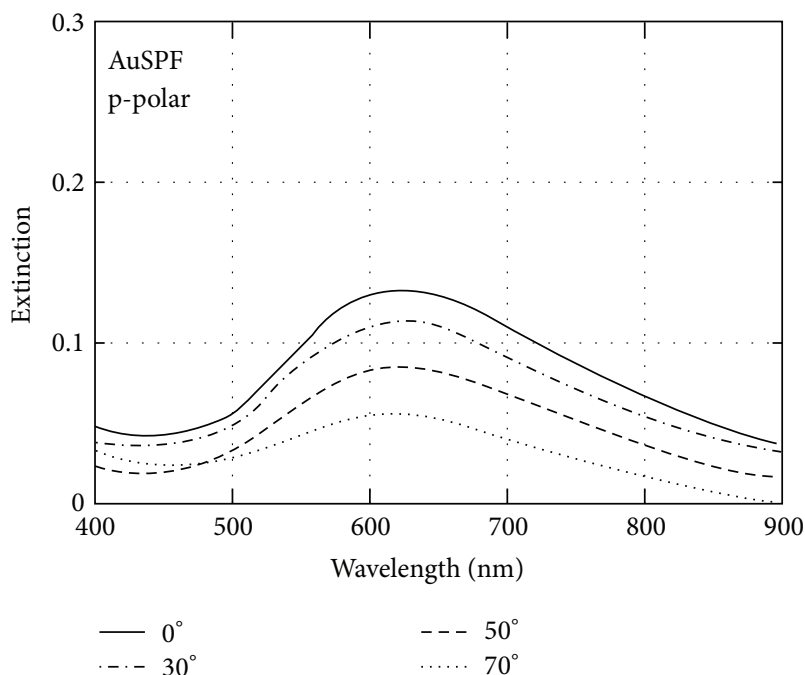

(c)

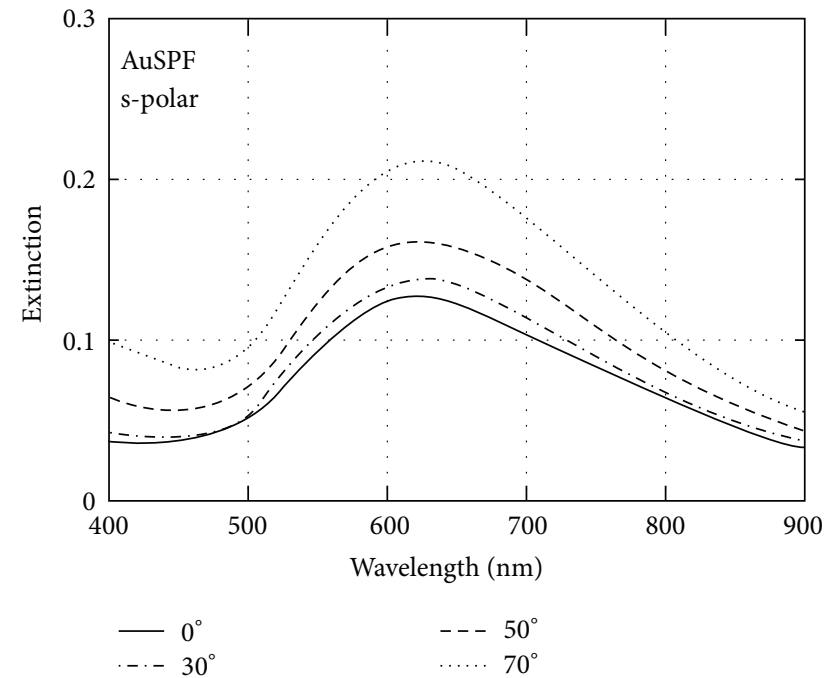

(d)

FIgURE 3: Polarized UV-visible extinction spectra of AuNF100 and AuSPF measured with variation in the angle of incidence between $\theta=0^{\circ}$ and $70^{\circ}$; (a) p-polarized and (b) s-polarized spectra of AuNF100; (c) p-polarized and (d) s-polarized spectra of AuSPF. The vertical axis is extinction (absolute). Inset in (a) is a high magnification of the spectra around a peak resonance wavelength.

TABLE 2: RI sensitivity and $\Delta \lambda_{\text {LPR }}$ of prepared samples.

\begin{tabular}{lcc}
\hline Sample & $\Delta \lambda_{\mathrm{LPR}}$ at $\theta=70^{\circ} / \mathrm{nm}$ & $\begin{array}{c}\mathrm{RI} \\
\text { sensitivity } / \mathrm{nmRIU}^{-1}\end{array}$ \\
\hline AuSPF & 7.8 & 227 \\
AuNF500 & 8.0 & 262 \\
AuNF200 & 22 & 446 \\
AuNF100 & 33 & 526 \\
Au nanospheres* & - & 20.3
\end{tabular}

${ }^{*} 20 \mathrm{~nm}$ diameter Au nanospheres deposited on a BK7 glass substrate.

of the thin nanofiber induces a curving and elongation of the Au nanoparticles at the nanofiber surface.
The polarization dependence of the LPR extinction intensity at $\lambda_{\mathrm{LPR}}$ supports the difference in the LPR characteristics of the Au nanoparticles in AuNF100, AuSPF, and the reference of the isotropic $20 \mathrm{~nm}$ diameter Au nanospheres (Tanaka Kikinzoku Kogyo K. K., Japan) chemically immobilized on a BK7 glass slide. Figure 4 summarizes the $\theta$ dependences of the LPR extinction intensity obtained from AuNF100, AuSPF, and the isotropic Au nanospheres. For the isotropic $\mathrm{Au}$ nanospheres, the extinction intensities in the p- and spolarized spectra showed almost the same incident angle dependence (see green solid circles and open squares in Figure 4). This is attributed to the isotropic shape of the $\mathrm{Au}$ nanosphere since the LPR extinction intensity related to the shape and the size of the Au nanoparticles. The increases in both extinction intensities are attributed to the increase in the 


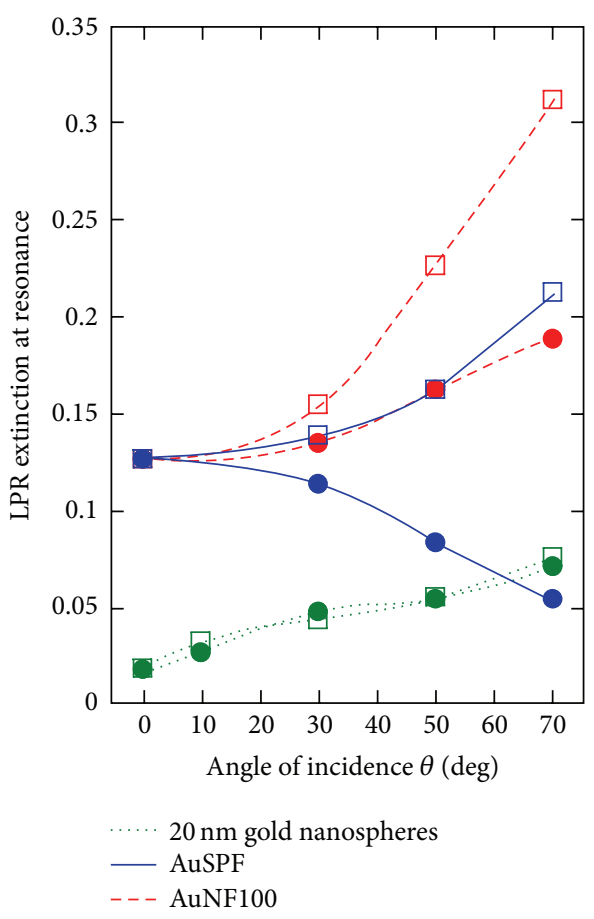

FIGURE 4: Incident angle dependences of LPR extinction intensity observed in AuNF100 (broken lines), AuSPF (solid lines), and $20 \mathrm{~nm}$ diameter Au nanospheres chemically immobilized on a BK7 glass substrate (dotted lines). For each sample, solid circle $(\bullet)$ and square ( $\square$ ) show the LPR extinctions in p-polarized and s-polarized extinction spectra, respectively. The lines are guides for the eye.

irradiated area on the sample surface. For AuSPF, in a series of p-polarized spectra, the extinction intensity decreased with the increase in $\theta$, while those in the s-polarized spectra increased (see blue solid circles and open squares). This polarization dependence of the extinction intensity agrees with that for the unannealed or annealed Au island structure on a dielectric substrate. Gupta et al. ascribed this polarization dependence to both the contribution from the shape of Au nanoparticles, which is truncated nanospheres or oblate, and the effect of the dielectric substrate to LPR [39-41]. For AuNF100, both the p- and s-polarized LPR extinctions increased with $\theta$, in which the s-polarized ones show a higher intensity than the p-polarized one at any angle (see red solid circles and open squares). The behavior of the extinction intensity in AuNF100 deviated from both the nanospheres and AuSPF indicates the different LPR characteristics, which may be because of not only the difference in the height but also the curved shape of the Au island structure in the AuNF100 and the effect of the dielectric nanofiber substrate.

LPRs in these Au nanoparticles prepared on a nanofiber showed considerably high RI sensitivities. In Figure 5(a), the transmittance extinction spectra of the AuNF100 immersed in RI liquids $(n=1.3330 \sim 1.4050)$ are shown. With an increase in the RI of the surroundings, the $\lambda_{\mathrm{LPR}}$ value is mostly redshifted and the RI sensitivity was found to be $526 \mathrm{~nm} / \mathrm{RIU}$. This sensitivity is higher than those of most anisotropic Au nanoparticles reported in the past, such as $\mathrm{Au}$ nanorods
(252 nm/RIU) [22-24] and Au nanotriangles (125 nm/RIU) [29]. It is comparable to the RI sensitivity of Au nanobipyramids ( $544 \mathrm{~nm} / \mathrm{RIU})$ [25] prepared by the reduction of $\mathrm{Au}$ nanospheres with a surface agent.

The RI sensitivities obtained from the prepared samples were enhanced with the reduction in the fiber diameter. Figure 5(b) plots the magnitude of the red-shift of $\lambda_{\text {LPR }}$ with variation of the RI in surroundings, $n$, for all the prepared samples. Both AuNF100 and AuNF200 had high RI sensitivities over $400 \mathrm{~nm} / \mathrm{RIU}$. On the other hand, AuNF500 and AuSPF showed $256 \mathrm{~nm} / \mathrm{RIU}$ and $227 \mathrm{~nm} / \mathrm{RIU}$ values, respectively, which are comparable to the sensitivity reported for annealed $\mathrm{Au}$ island films on ITO glass (217 nm/RIU at maximum) [32]. This behavior of the RI sensitivity depending on the mean fiber diameters is similar to that of $\Delta \lambda_{\text {LPR }}$ as summarized in Table 2, which suggests an interrelation between the improvement of the RI sensitivity and the aspect ratio of the Au nanoparticles. Both RI sensitivities and $\Delta \lambda_{\text {LPR }}$ showed higher values in AuNF200 and AuNF100 compared with others. It appears to be that there is a certain criterion for high RI sensitivity between AuNF200 and AuNF500. Based on this finding, we concluded that improvement of the RI sensitivity was mainly due to variation of the shape of Au nanoparticle with large contrast in the surface curvature of the nanofibers though the effect from the substrate, the dielectric nanofiber in this case, also might contribute to the LPR characteristics as already reported by theoretical studies [39-42].

To quantitatively understand the origin of this great improvement of the RI sensitivity, three-dimensional observations of the prepared Au nanoparticles by transmission electron microscopy and theoretical calculation of the LPR extinction for the obtained shape on the nanofibers will be required. The relationship between the shape of the $\mathrm{Au}$ nanoparticle and the RI sensitivity will be more comprehensive when the prepared Au nanoparticles annealed at over $200^{\circ} \mathrm{C}$ that will change their shape to truncated spheroids on the nanofibers if the nanofiber morphologies were kept during annealing. In addition, we found that the nanofiber thinner than $50 \mathrm{~nm}$ incidentally formed in AuNF200 and AuNF100 with one or two Au nanoparticles on their width as shown in the Supporting Information, available online at http://dx.doi.org/10.1155/2015/829273 (Figure S1). We believe that if we could prepare the substrate consisting of ultrathin nanofibers whose diameters were nearly equal to the size of the $\mathrm{Au}$ nanoparticles like these fibers, the LPRs in them may provide a dramatic improvement in the RI sensitivity or interesting new aspects of the LPR.

The prepared Au nanoparticles showed no variation in the LPR extinction during the extinction measurement in a RI liquid and did not peel off from the nanofiber surface (the Au nanoparticles evaporated on glass substrate without adhesion by $\mathrm{Cr}$ or Ti easily peel off in various solvents, including water). This is also an advantage of our anisotropic Au nanoparticles with exception of high RI sensitivity. The hierarchically structured LPR sensing fibers provide the good handling characteristics and flexibility to be processed in diverse forms (e.g., LPR sensing fabrics) for practical use. 


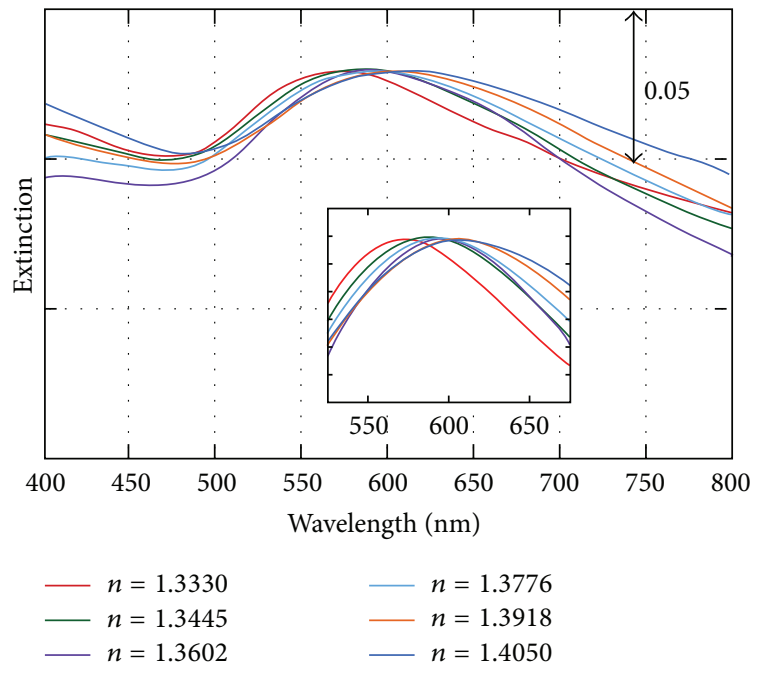

(a)

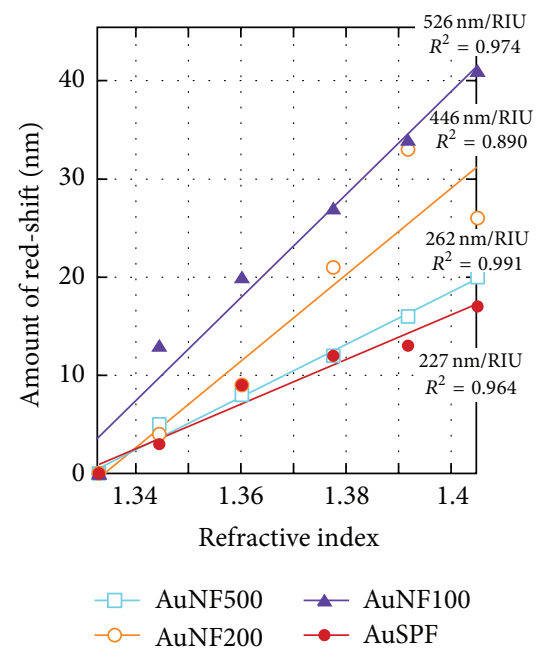

(b)

FIGURE 5: (a) Extinction spectra of the AuNF100 in various RI liquids. (b) The magnitude of red-shifting of LSP extinction of prepared samples with respect to refractive index of surroundings. The slope of the plot corresponds to the RI sensitivity.

\section{Conclusion}

This paper reported the facile and high-throughput fabrication method of anisotropic curved Au nanoparticles with a highly sensitive LPR using the cylindrical surface of electrospun nanofibers. SEM observations and polarized extinction spectroscopy revealed the formation of the curved Au islands on the nanofibers. The fiber diameter dependence of the anisotropy and the RI sensitivity of their LPRs demonstrated an enhancement in the RI sensitivity due to anisotropy of the $\mathrm{Au}$ nanoparticles based on the large contrast in the surface curvature of the nanofibers. The Au nanoparticledecorated nanofiber showed a RI sensitivity of $526 \mathrm{~nm} / \mathrm{RIU}$ at maximum for $\sim 100 \mathrm{~nm}$ thick nanofibers $(\phi=114 \mathrm{~nm})$, which is higher than what most LPR materials reported in the past. The anisotropic Au nanoparticle-decorated nanofiber or nanofibrous fabrics fabricated by the high-throughput process provide a promising platform with high-sensitivity for the label-free sensing of biomaterials. In addition, it also provides a promising option for surface enhanced Raman scattering spectroscopy. The study on sensing practical biomaterials and enhancement of Raman scattering using our materials will be reported in the future.

\section{Conflict of Interests}

The authors declare that there is no conflict of interests regarding the publication of this paper.

\section{Acknowledgments}

The authors thank the New Energy Development Organization (NEDO), Japan, for the funding. The authors also thank Mr. Jun Koki, Center for Advanced Materials Analysis, Tokyo
Institute of Technology, for the SEM observation of the Au nanoparticles.

\section{References}

[1] C. Y. Chen and E. Burstein, "Giant Raman scattering by molecules at metal-island films," Physical Review Letters, vol. 45, no. 15, pp. 1287-1291, 1980.

[2] K. Kneipp, Y. Wang, H. Kneipp et al., "Single molecule detection using surface-enhanced Raman scattering (SERS)," Physical Review Letters, vol. 78, no. 9, pp. 1667-1670, 1997.

[3] N. Félidj, J. Aubard, G. Lévi et al., "Optimized surface-enhanced Raman scattering on gold nanoparticle arrays," Applied Physics Letters, vol. 82, no. 18, pp. 3095-3097, 2003.

[4] W. Zhang, H. Fischer, T. Schmid, R. Zenobi, and O. J. F. Martin, "Mode-selective surface-enhanced Raman spectroscopy using nanofabricated plasmonic dipole antennas," The Journal of Physical Chemistry C, vol. 113, no. 33, pp. 14672-14675, 2009.

[5] J. Jiang, K. Bosnick, M. Maillard, and L. Brus, "Single molecule Raman spectroscopy at the junctions of large Ag nanocrystals," The Journal of Physical Chemistry B, vol. 107, no. 37, pp. 99649972, 2003.

[6] R. Bardhan, N. K. Grady, and N. J. Halas, "Nanoscale control of near-infrared fluorescence enhancement using au nanoshells," Small, vol. 4, no. 10, pp. 1716-1722, 2008.

[7] T. Nakamura and S. Hayashi, "Enhancement of dye fluorescence by gold nanoparticles: analysis of particle size dependence," Japanese Journal of Applied Physics, vol. 44, no. 9, pp. 6833-6837, 2005.

[8] K. Tsuboi, S. Abe, S. Fukuba et al., "Second-harmonic spectroscopy of surface immobilized gold nanospheres above a gold surface supported by self-assembled monolayers," The Journal of Chemical Physics, vol. 125, no. 17, Article ID 174703, 2006.

[9] J. Homola, S. S. Yee, and G. Gauglitz, "Surface plasmon resonance sensors: review," Sensors and Actuators, B: Chemical, vol. 54, no. 1, pp. 3-15, 1999. 
[10] C. R. Yonzon, D. A. Stuart, X. Zhang, A. D. McFarland, C. L. Haynes, and R. P. van Duyne, "Towards advanced chemical and biological nanosensors-an overview," Talanta, vol. 67, no. 3, pp. 438-448, 2005.

[11] J. N. Anker, W. P. Hall, O. Lyandres, N. C. Shah, J. Zhao, and R. P. Van Duyne, "Biosensing with plasmonic nanosensors," Nature Materials, vol. 7, no. 6, pp. 442-453, 2008.

[12] S.-Y. Fukuba, K. Tsuboi, S. Abe, and K. Kajikawa, "Nonlinear optical detection of proteins based on localized surface plasmons in surface immobilized gold nanospheres," Langmuir, vol. 24, no. 15, pp. 8367-8372, 2008.

[13] J. S. Yuk, H.-S. Kim, J.-W. Jung et al., "Analysis of protein interactions on protein arrays by a novel spectral surface plasmon resonance imaging," Biosensors and Bioelectronics, vol. 21, no. 8, pp. 1521-1528, 2006.

[14] T. Okamoto, I. Yamaguchi, and T. Kobayashi, "Local plasmon sensor with gold colloid monolayers deposited upon glass substrates," Optics Letters, vol. 25, no. 6, pp. 372-374, 2000.

[15] R. Tanaka, T. Yuhi, N. Nagatani et al., "A novel enhancement assay for immunochromatographic test strips using gold nanoparticles," Analytical and Bioanalytical Chemistry, vol. 385, no. 8, pp. 1414-1420, 2006.

[16] K. Mitsui, Y. Handa, and K. Kajikawa, "Optical fiber affinity biosensor based on localized surface plasmon resonance," Applied Physics Letters, vol. 85, no. 18, pp. 4231-4233, 2004.

[17] K. Tsuboi, S. Fukuba, R. Naraoka, K. Fujita, and K. Kajikawa, "Multichannel biosensing platform of surface-immobilized gold nanospheres for linear and nonlinear optical imaging," Applied Optics, vol. 46, no. 20, pp. 4486-4490, 2007.

[18] A. D. McFarland and R. P. van Duyne, "Single silver nanoparticles as real-time optical sensors with zeptomole sensitivity," Nano Letters, vol. 3, no. 8, pp. 1057-1062, 2003.

[19] J. J. Mock, D. R. Smith, and S. Schultz, "Local refractive index dependence of plasmon resonance spectra from individual nanoparticles," Nano Letters, vol. 3, no. 4, pp. 485-491, 2003.

[20] S. M. Marinakos, S. Chen, and A. Chilkoti, "Plasmonic detection of a model analyte in serum by a gold nanorod sensor," Analytical Chemistry, vol. 79, no. 14, pp. 5278-5283, 2007.

[21] W. Cai, T. Gao, H. Hong, and J. Sun, "Applications of gold nanoparticles in cancer nanotechnology," Nanotechnology, Science and Applications, vol. 2008, no. 1, pp. 17-32, 2008.

[22] K. L. Kelly, E. Coronado, L. L. Zhao, and G. C. Schatz, "The optical properties of metal nanoparticles: the influence of size, shape, and dielectric environment," The Journal of Physical Chemistry B, vol. 107, no. 3, pp. 668-677, 2003.

[23] Y. Xia and N. J. Halas, "Shape-controlled synthesis and surface plasmonic properties of metallic nanostructures," MRS Bulletin, vol. 30, no. 5, pp. 338-348, 2005.

[24] P. Kvasnička and J. Homola, "Optical sensors based on spectroscopy of localized surface plasmons on metallic nanoparticles: sensitivity considerations," Biointerphases, vol. 3, no. 3, pp. FD4-FD11, 2008.

[25] H. Chen, X. Kou, Z. Yang, W. Ni, and J. Wang, "Shape- and sizedependent refractive index sensitivity of gold nanoparticles," Langmuir, vol. 24, no. 10, pp. 5233-5237, 2008.

[26] C. L. Nehl, H. Liao, and J. H. Hafner, "Optical properties of starshaped gold nanoparticles," Nano Letters, vol. 6, no. 4, pp. 683688, 2006.

[27] F. Hao, Y. Sonnefraud, P. van Dorpe, S. A. Maier, N. J. Halas, and P. Nordlander, "Symmetry breaking in plasmonic nanocavities: Subradiant LSPR sensing and a tunable Fano resonance," Nano Letters, vol. 8, no. 11, pp. 3983-3988, 2008.
[28] M. Himmelhaus and H. Takei, "Cap-shaped gold nanoparticles for an optical biosensor," Sensors and Actuators B: Chemical, vol. 63, no. 1, pp. 24-30, 2000.

[29] A. J. Haes and R. P. van Duyne, "A nanoscale optical biosensor: sensitivity and selectivity of an approach based on the localized surface plasmon resonance spectroscopy of triangular silver nanoparticles," Journal of the American Chemical Society, vol. 124, no. 35, pp. 10596-10604, 2002.

[30] D. Jia and A. Goonewardene, "Two-dimensional nanotriangle and nanoring arrays on silicon wafer," Applied Physics Letters, vol. 88, no. 5, Article ID 053105, pp. 1-3, 2006.

[31] A. Unger, U. Rietzler, R. Berger, and M. Kreiter, "Sensitivity of crescent-shaped metal nanoparticles to attachment of dielectric colloids," Nano Letters, vol. 9, no. 6, pp. 2311-2315, 2009.

[32] S. Szunerits, V. G. Praig, M. Manesse, and R. Boukherroub, "Gold island films on indium tin oxide for localized surface plasmon sensing," Nanotechnology, vol. 19, no. 19, Article ID 195712, 2008.

[33] J. Doshi and D. H. Reneker, "Electrospinning process and applications of electrospun fibers," Journal of Electrostatics, vol. 35, no. 2-3, pp. 151-160, 1995.

[34] A. Greiner and J. H. Wendorff, "Electrospinning: a fascinating method for the preparation of ultrathin fibers," Angewandte Chemie, vol. 46, no. 30, pp. 5670-5703, 2007.

[35] H. Kuwayama, H. Matsumoto, K. Morota, M. Minagawa, and A. Tanioka, "Control over color of nanotextured coatings by electrospray deposition," Sen'i Gakkaishi, vol. 64, no. 1, pp. 1-4, 2008.

[36] H. Matsumoto and A. Tanioka, "Functionality in electrospun nanofibrous membranes based on fiber's size, surface area, and molecular orientation," Membranes, vol. 1, no. 3, pp. 249-264, 2011.

[37] M. Nasir, H. Matsumoto, T. Danno et al., "Control of diameter, morphology, and structure of PVDF nanofiber fabricated by electrospray deposition," Journal of Polymer Science, Part B: Polymer Physics, vol. 44, no. 5, pp. 779-786, 2006.

[38] K. Nakashima, K. Tsuboi, H. Matsumoto et al., "Control over internal structure of liquid crystal polymer nanofibers by electrospinning," Macromolecular Rapid Communications, vol. 31, no. 18, pp. 1641-1645, 2010.

[39] R. J. Warmack and S. L. Humphrey, "Observation of two surface-plasmon modes on gold particles," Physical Review B, vol. 34, no. 4, pp. 2246-2252, 1986.

[40] G. Gupta, D. Tanaka, Y. Ito et al., "Absorption spectroscopy of gold nanoisland films: optical and structural characterization," Nanotechnology, vol. 20, no. 2, Article ID 025703, 2009.

[41] M. M. Wind, J. Vlieger, and D. Bedeaux, "The polarizability of a truncated sphere on a substrate I," Physica A: Statistical Mechanics and its Applications, vol. 141, no. 1, pp. 33-57, 1987.

[42] R. Chauvaux and A. Meessen, "Multipole oscillations in small hemispherical particles," Thin Solid Films, vol. 62, no. 1, pp. 125131, 1979. 

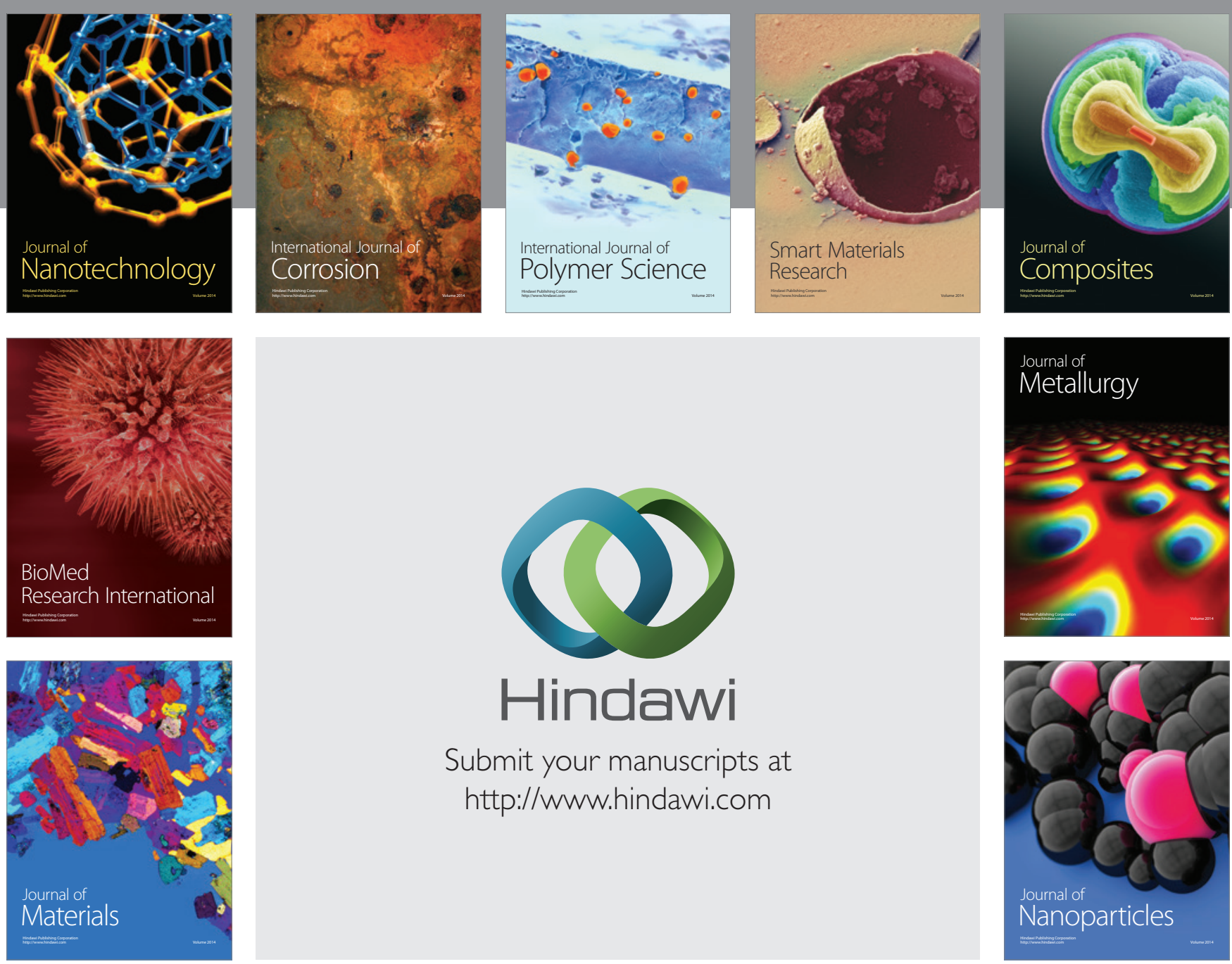

Submit your manuscripts at http://www.hindawi.com
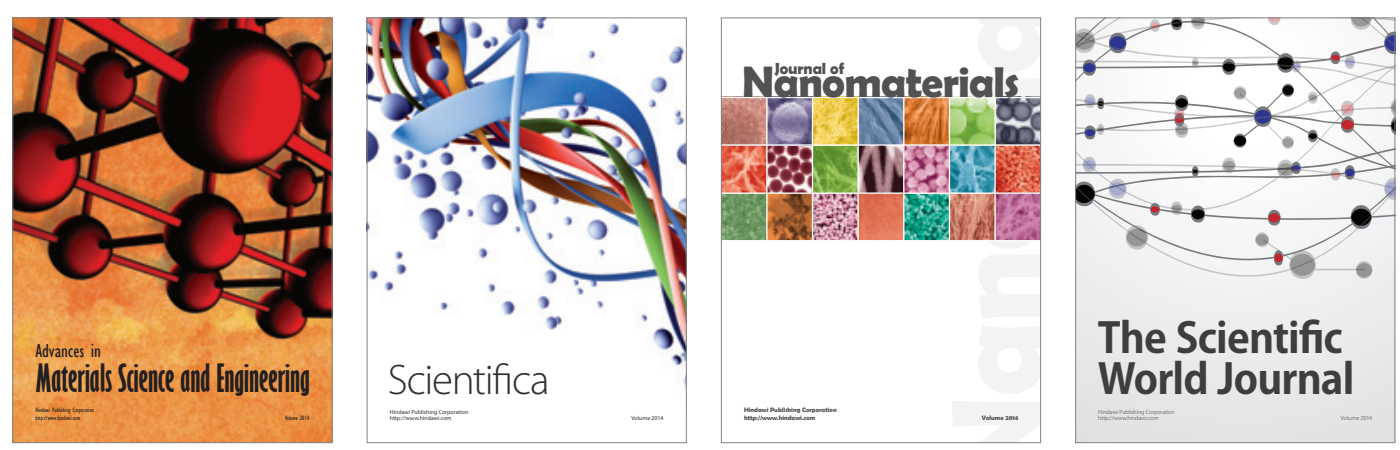

\section{The Scientific World Journal}
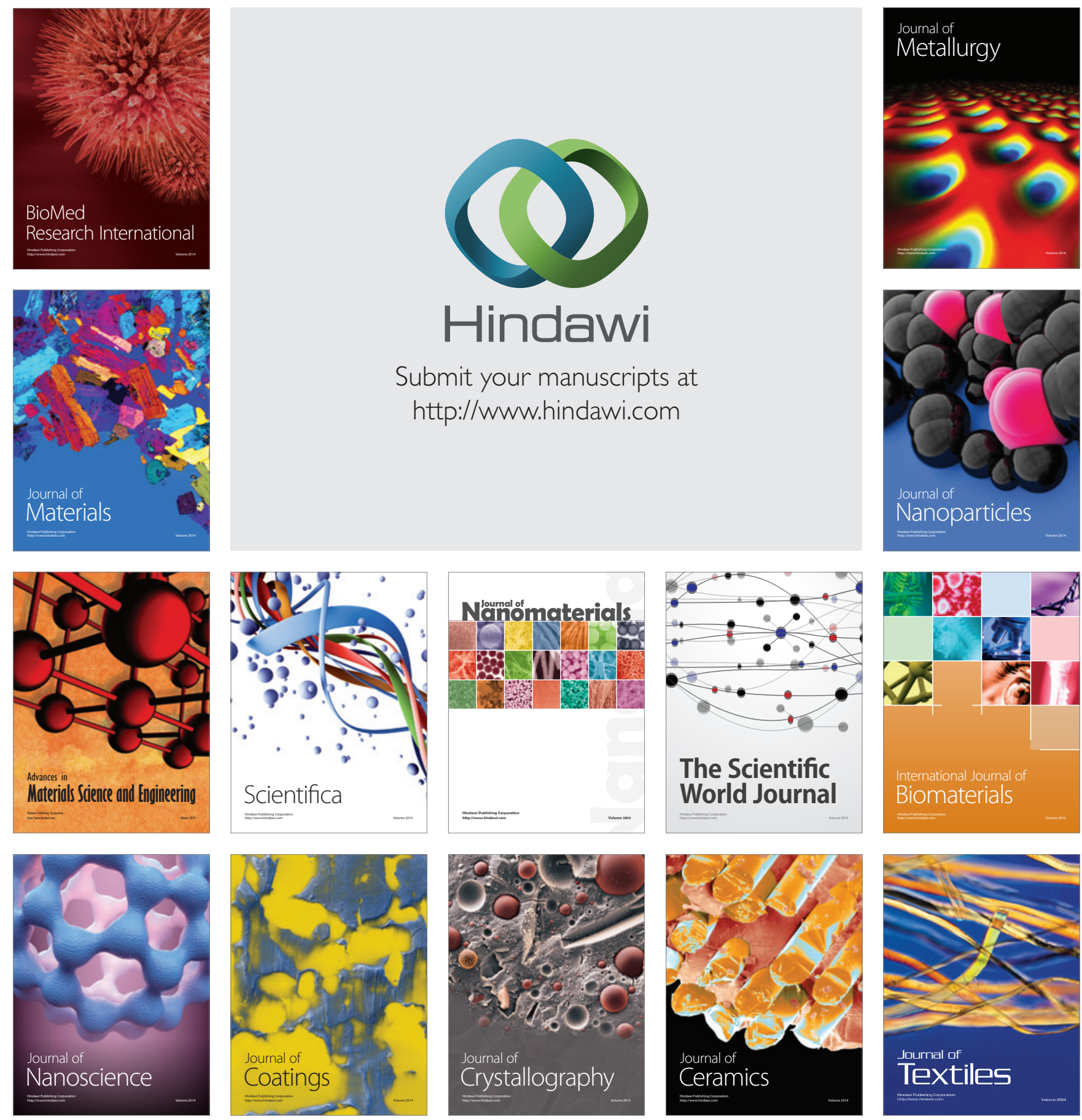